\title{
Artículos
}

\section{La impulsividad, la ira y las estrategias de poder como predictores de la violencia de pareja, con}

\author{
mujeres perpetradoras
}

\section{Impulsiveness, anger and power strategies as predictors of partner violence with female perpetrators}

\author{
Manuel Alejandro Galindo Moto; Cinthia Cruz del Castillo; Rubén Esteban Lechuga Paredes; Rolando Díaz- \\ Loving ${ }^{2}$;Angélica Romero Palencia ${ }^{3}$; Joaquín Alberto Padilla Bautista ${ }^{4}$
}

1 Universidad Iberoamericana

2 Universidad Nacional Autónoma de México

3 Universidad Autónoma del Estado de Hidalgo

4 Universidad Autónoma de Baja California

\section{Resumen}

La ira, la impulsividad y las relaciones de poder han sido señaladas como causales de la violencia de pareja. Estos predictores se han estudiado de manera independiente y considerando la violencia física ejercida por hombres. Por esta razón, el propósito de esta investigación fue estudiar las variables como predictores en conjunto y diferenciar su efecto en la violencia psicológica, física, económica, social y sexual, en mujeres perpetradoras. El estudio se llevó a cabo en una muestra no probabilística de 532 mujeres $(X=26.27)$. Para cubrir tal propósito se llevaron a cabo regresiones múltiples y simples. Como predictores de la violencia psicológica/física se utilizaron los factores de manipulación y chantaje, el dominio, la reacción de la ira, el automatismo, la gratificación y el temperamento iracundo, se obtuvo una $\mathrm{R}^{2} .40$. Como predictores de la violencia económica/social se utilizaron los factores de manipulación y chantaje, la reacción de ira, el dominio, la gratificación y el automatismo, obteniendo una $\mathrm{R}^{2}$.19. Para predecir la violencia sexual se realizaron regresiones simples, sin embargo, la varianza explicada por cada una de las variables fue muy baja. Se sugiere que la violencia física, psicológica, económica y social comparten las mismas causas.

Palabras clave: ira, impulsividad, violencia de pareja, mujeres violentas, estrategias de poder

1 Manuel Alejandro Galindo Moto, Universidad Iberoamericana.

1 Cinthia Cruz del Castillo Universidad Iberoamericana

1 Rubén Esteban Lechuga Paredes Universidad Iberoamericana.

2 Rolando Díaz-Loving Universidad Nacional Autónoma de México

3 Angélica Romero Palencia Universidad Autónoma del Estado de Hidalgo

4 Joaquín Alberto Padilla Bautista Universidad Autónoma de Baja California

Autor para correspondencia: psicogalindo@live.com.mx 


\begin{abstract}
Anger, impulsiveness, and power relationships have been identified as causes of intimate partner violence. These predictors have been studied independently and exclusively considering physical violence by men. For this reason, the purpose of this research was to study the variables as predictors and to differentiate their effect on psychological, physical, economic, social, and sexual violence in female perpetrators. The study was carried out in a non-probabilistic sample of 532 women $(X=26.27)$. To accomplish this purpose, multiple stepwise regressions and simple regressions were carried out. As predictors of psychological / physical violence, the following factors were used: manipulation and blackmail, dominance, anger reaction, automatism, gratification, and angry temperament, obtaining an $\mathrm{R}^{2} .40$. As predictors of economic / social violence, the following factors were used: manipulation and blackmail, the reaction of anger, dominance, gratification, and automatism, obtaining an $\mathrm{R}^{2} .19$. Simple regressions were carried out to predict sexual violence, however the variance explained by each of the variables was very low. It is suggested that physical, psychological, economic, and social violence share the same causes.
\end{abstract}

Key words: Anger, impulsiveness, partner violence, violent women, power strategies

DOI https://doi.org/10.36793/psicumex.v11i1.357

Recibido 30 de mayo de 2020

Aceptado 29 de abril de 2021

Publicado 2 de junio de 2021 


\section{Introducción}

Se ha investigado a la violencia de pareja por más de 50 años (Nicolson, 2019). A pesar de esto, Conradi y Geffner (2012) han propuesto estudiar el fenómeno con las mujeres como perpetradoras, así como estudiar las diferencias entre los distintos tipos de violencia de pareja, debido a que no hay suficientes investigaciones al respecto.

La postura feminista fue de las primeras teorías en ofrecer modelos explicativos de la violencia de pareja, por lo que se ha producido mucha más investigación con su marco teórico (Archer, 2000; Nicolson, 2019). La postura feminista suele dejar de lado la violencia con la mujer como perpetradora, particularmente la violencia económica, la violencia social y la violencia sexual. Snyder et al. (2018) en una antología de investigaciones de violencia de pareja de los últimos 10 años omiten casi por completo la violencia con la mujer como perpetradora y de manera absoluta la violencia de pareja no física. Primero es importante señalar que la violencia de pareja se explica cómo todo comportamiento en el que un miembro de la pareja cause daño o tenga la intención de dañar a su pareja (Díaz-Loving y Rivera, 2010; Lien y Lorentzen, 2019; Nicolson, 2019).

Existen cinco tipos de violencia de pareja: violencia física, violencia social, violencia económica, violencia sexual y violencia psicológica. La violencia física consiste en lastimar o tener la intención de lastimar físicamente a la pareja (Díaz-Loving y Rivera, 2010; Lien y Lorentzen, 2019; Organización Mundial de la Salud, 2016). La violencia psicológica consiste en que un miembro de la pareja lastime emocionalmente a su pareja (Díaz-Loving y Rivera, 2010; Lien y Lorentzen, 2019; OMS, 2016). La violencia económica se describe como el control de los recursos financieros, aun a pesar de las necesidades personales, por parte de un miembro de la pareja sobre el otro/a (Díaz-Loving y Rivera, 2010; Nicolson, 2019; OMS, 2016). La violencia social consiste en controlar las relaciones sociales de la pareja (Díaz-Loving y Rivera, 2010). Por 
último, la violencia sexual se describe como los comportamientos sexuales en contra de la voluntad de la pareja (Díaz-Loving y Rivera, 2010; OMS, 2016; Rivera et al., 2017).

La prevalencia de los cinco tipos de violencia de pareja varía de acuerdo con distintas investigaciones, la violencia psicológica es la más prevalente (Fernández et al., 2016; Sears et al., 2007; Siller et al., 2012). Por ejemplo, Williams et al. (2008) encontraron que eran entre el $81 \%$ y el $88 \%$ de las mujeres las que ejercían violencia psicológica. En cuanto a la violencia física, los mismos autores señalaron que los porcentajes varían dependiendo de si son grupos considerados de alto riesgo y de la gravedad de la violencia ejercida, por lo que señalan porcentajes entre el $13 \%$ y el $68 \%$ (Williams et al., 2008).

En la Ciudad de México, Siller et al. (2012) encontraron que el $5.8 \%$ de las mujeres han ejercido este tipo de violencia, mientras que en la ciudad de Tijuana se encontró que el $33.10 \%$ de las mujeres han ejercido la violencia física (Fernández et al., 2016). Por otra parte, Siller et al. (2012) encontraron que las mujeres que ejercen la violencia social son alrededor del 15.2 \%. En cuanto a la violencia económica, en la Ciudad de México, Siller et al. (2012) encontraron que el $15.5 \%$ de las mujeres han ejercido este tipo de violencia. En cambio, en Tijuana, la prevalencia fue del $8 \%$ para perpetradoras (Fernández et al., 2016).

La violencia sexual es la menos prevalente de las tres. En mujeres adolescentes perpetradoras llega al $5 \%$ de acuerdo a Sears et al. (2007), mientras que Williams et al. (2008) encontraron que la prevalencia estuvo entre el $4.6 \%$ y el $19.5 \%$, dependiendo de la investigación. En México se encontraron resultados similares con mujeres perpetradoras, por ejemplo, en la Ciudad de México la prevalencia fue de $6.9 \%$, mientras que en Tijuana fue de 3.10 \% (Fernández et al., 2016; Siller et al., 2012).

Los modelos teóricos utilizados para explicar la violencia de pareja hacen énfasis en el aprendizaje de dinámicas violentas, como lo puede ser la teoría de Bandura del aprendizaje social que señala que la violencia es aprendida y que las dinámicas de pareja recrean dicho aprendizaje (Bandura, 1978; Dutton, 2006). Otros autores como Dutton (2006) o Díaz-Loving y Rivera (2010) retoman a Bronfenbrenner, que 
sugiere que se aprende a ser violento en los distintos ambientes sociales en los que se desarrolla el individuo. Esta postura sostiene que se aprenden creencias y medios de solución violentos, además de que se desarrollan rasgos de personalidad o psicopatologías que facilitan los comportamientos violentos en la pareja. Esto implica que es en el proceso de desarrollo en el que se aprenden comportamientos que llevan a la violencia o que la falta de desarrollo de factores de protección ante la violencia facilita que esta ocurra.

De acuerdo con los modelos mencionados previamente, las perpetradoras de violencia de pareja tienen guiones agresivos o respuestas aprendidas que facilitan la violencia. El ejercicio de poder basado en el control ha sido señalado como un comportamiento aprendido en la infancia que se manifiesta en las relaciones de pareja (Bewley y Welch, 2014; Dutton, 2006). Para algunos autores la necesidad de controlar a la pareja es lo que lleva a la violencia física (Bewley y Welch, 2014; Dutton, 2007; Johnson, 2008; Stark y Hester, 2019). En cambio, para otros autores, cuando disminuye el poder del perpetrador/a, se desata la violencia para recuperar el control perdido (Frankland y Brown, 2014; Resko, 2010). De acuerdo con diversos autores, es a través de la masculinidad tóxica que el hombre tiene una necesidad de controlar a la mujer (Bewley y Welch, 2014; Johnson, 2008); sin embargo, esta necesidad de dominar a la pareja no se ha encontrado solamente en los hombres, también se ha encontrado en las mujeres y en relaciones no violentas.

Por ejemplo, las estrategias de poder son los métodos con los cuales un individuo busca influir en su pareja, estas pueden ser positivas o negativas y se consideran negativas cuando implican el castigo como medio de control (Rivera y Díaz-Loving, 2002; Rivera et al., 2017). Las estrategias de poder negativas son las siguientes: coerción, en la que se utiliza la violencia física y las amenazas; la estrategia de poder de manipulación y chantaje, en la que se intenta influir a través de la culpa, y la estrategia de poder de dominio, en la que el sujeto castiga para obtener lo que quiere (Rivera y Díaz-Loving, 2002; Rivera et al., 2017). 
Aun así, la capacidad de autorregularse puede determinar que las luchas de poder en la pareja no se vuelvan violentas. Celma (2015) señala que la habilidad de autorregularse se desarrolla y aprende durante la niñez. Si no hay reflexión o autorregulación, las respuestas pueden ser impulsivas y violentas en la pareja.

La impulsividad es una falta de control de las emociones y comportamientos. Implica ser irreflexivo y tener dificultad para controlar la respuesta a los estímulos que se perciben (Alcázar-Córcoles et al., 2015; Celma, 2015; Salvo y Castro, 2013). La impulsividad tiene tres manifestaciones: 1) gratificación, es la incapacidad para retrasar una recompensa inmediata; 2) automatismo, es la repetición de una conducta a pesar de no obtener un refuerzo o de recibir castigo; 3 ) atencional, es dar respuestas rápidas sin contar con toda la información necesaria (Iribarren, et al., 2011).

De acuerdo con López (2013), la impulsividad se puede relacionar en un contexto determinado, como la pareja, con una respuesta problemática, que es la agresión. Y es que las personas impulsivas no planean bien sus acciones y reaccionan con violencia, además de que no tienen control durante los conflictos (McMurran et al., 2002; Teten et al., 2012; Thornton et al., 2016).

Por otra parte, Dutton (2007) y Potter-Efron (2015) señalan que la ira también facilita que ocurra la violencia de pareja. Dutton y Golant (2012) mencionan que la respuesta de ira se aprende al ver la violencia en la infancia, lo que provoca una necesidad de control que se manifiesta en las relaciones de pareja. Adicionalmente, Potter-Efron (2015) con una perspectiva ontogenética señala que la ira es una experiencia que se desarrolla a partir de eventos estresantes y al vivir violencia en la infancia, por lo que se aprende a responder con ira.

Autores como Nedegaard y Sbrocco (2014) han generado evidencia de que la ira favorece la violencia de pareja porque perjudica la toma de decisiones y se internalizan emociones negativas que se manifiestan en hostilidad (Birkley y Eckhardt, 2015). Sin embargo, la ira por sí misma no es buena o mala, para Elias y Saucier (2006) la ira es una emoción básica, intrínseca a la condición humana, un atavismo evolutivo y una 
adaptación filogenética que prepara para la agresión. La ira se puede manifestar como rasgo, como estado y puede ser controlada o expresada (Oliva et al., 2010; Potter-Efron, 2015). La ira como rasgo se refiere al cómo se vive la ira a lo largo del tiempo y se divide en:

a) Temperamento iracundo, que se refiere a vivir ira de manera continua.

b) Reacción de ira, que se refiere a qué tan frecuente se responde con ira.

La ira como estado es una respuesta psicoemocional que señala la intensidad de los sentimientos y las ganas de expresarlos, se divide en:

a) Deseos de expresar ira, que es tener el deseo emocional de manifestar la ira.

b) Sentimientos de ira, que es tener emociones de ira de manera continua.

Esta clasificación puede señalar que favorece más la violencia: la ira como rasgo o como estado. Es decir, la ira como rasgo describe lo que es vivir con ira; mientras que la ira como estado, el reaccionar emocionalmente con ira. También se puede utilizar la clasificación de la impulsividad de Iribarren, et al. (2011) para conocer qué manifestación de la impulsividad se relaciona más con la violencia de pareja, por ejemplo, si es la atención, el automatismo o la gratificación. La evidencia previa señala que el automatismo es una característica de las personas violentas, sin embargo, se desconoce si la gratificación o la atención se relacionan con la violencia de pareja.

Por otra parte, autores como Johnson (2008) o Bewley y Welch (2014) señalan que los hombres que son agresores suelen buscar el control. Aun así, el mismo Johnson (2008) y Bair-Merritt et al. (2010) han mencionado que la violencia por control también ocurre con las mujeres como perpetradoras, lo cual se contrapone con la perspectiva de género de la violencia de pareja, puesto que implica que las mujeres también tienen una necesidad de poder-control. Sin embargo, es importante revisar si las estrategias de poder de Rivera et al. (2017) se pueden utilizar para explicar la violencia de pareja. Estos planteamientos llevan a 
la necesidad de estudiar qué características de la ira y la impulsividad facilitan más la violencia de pareja, además de estudiar las estrategias de poder con las mujeres como perpetradoras.

Con base en lo anterior, el objetivo de este trabajo fue relacionar la ira, la impulsividad y a las estrategias de poder con la violencia psicológica/física, la violencia económica/social y la violencia sexual. Se hipotetizan relaciones positivas entre todas las variables, así como que tanto la ira y la impulsividad, como las estrategias de poder, predecirán los diferentes tipos de violencia. Así también, se hipotetiza que existirán diferencias significativas en la ira, la impulsividad y las estrategias de poder dependiendo del nivel de violencia ejercida por las mujeres.

\section{Metodología}

\section{Objetivo}

El objetivo de esta investigación fue generar un modelo predictor para la violencia económica, la violencia social, la violencia sexual, la violencia psicológica y la violencia física perpetrada por las mujeres. Es una investigación cuantitativa, no experimental, de tipo transversal y explicativa. Para cumplir el objetivo se utilizaron variables asociadas a la violencia física perpetrada por los hombres: estrategias de poder, impulsividad e ira.

\section{Participantes}

Es una muestra no probabilística, accidental, obtenida en la ciudad de Puebla. Se encuestó a las mujeres que se encontraban en lugares públicos como el zócalo, plazas comerciales y la central de camiones. Se eligieron lugares que fueran altamente frecuentados por personas de todas las edades y clases sociales. Las participantes son 532 mujeres de entre 18 y 60 años cuya media de edad fue de 26.27 años con una desviación estándar de 9.67.

De la muestra obtenida, el $33.5 \%$ no tenía pareja al momento de ser encuestada, el $50.1 \%$ tenía algún tipo de relación de noviazgo, el $14.1 \%$ se encontraba casada, mientras que el $2.3 \%$ no especificó su 
estatus de pareja. Al retomar datos de las mujeres que no tenían pareja en ese momento, se obtuvo información de relaciones previas. Se excluyó a los hombres, a las mujeres mayores de 60 años y a las adolescentes menores de 18 años; esto se debió a que los adolescentes suelen tener relaciones de pareja más breves o no tener relaciones de pareja alguna. Por su parte, los adultos mayores vivieron relaciones de pareja con paradigmas de género tradicionales, por lo que resulta importante estudiarlos de manera separada. Para asegurar que la cantidad de participantes fuera suficiente, se realizaron evaluaciones de potencia estadística, por lo que se describen los datos con potencia mayor a .80 .

\section{Instrumentos}

Se trabajó con cuatro instrumentos. La primera escala utilizada fue la de violencia de pareja perpetrador/a y receptor/a, cuya autora es Cienfuegos (2014). Solamente se trabajó con los factores de nivel perpetrador que incluyen los factores de violencia psicológica/física, económica/social y sexual. De esta manera se puede contrastar los diferentes tipos de violencia de pareja y compararlos entre sí.

En cuanto a la violencia sexual consiste en un solo reactivo. El alfa de Cronbach global del apartado de violencia de pareja de nivel perpetrador que proporcionó la autora Cienfuegos (2014), fue de $\alpha=.84$. Para asegurar la confiabilidad del instrumento se realizaron evaluaciones de alfa de Cronbach por factor, en las que se encontró un $\alpha=.74$ en violencia psicológica/física, mientras que en violencia económica/social fue $\alpha$ $=.68$. Finalmente, se señala que el indicador de violencia sexual no tiene alfa de Cronbach debido a que es solamente un reactivo. Las respuestas de los reactivos en la escala de Cienfuegos (2014) van del uno al cinco.

La segunda escala utilizada fue la de las estrategias de poder, cuyos autores son Rivera, Díaz-Loving y García (Rivera et al., 2017). Esta escala cuenta con 5 factores: manipulación y chantaje, dominio, coerción, equidad/racional y recompensa. Sin embargo, solamente se laboró con los factores de manipulación y chantaje y dominio, debido a que los modelos de violencia de pareja de Johnson (2008) y Dutton (2007) 
describen un ejercicio de poder de control en la violencia de pareja, lo que coincide teóricamente con los factores de dominio y manipulación y chantaje.

El factor de coerción también es omitido debido a que es definido por Rivera et al. (2017) como una estrategia de poder que ya mide la violencia física. Se eligieron los factores que representan las estrategias de poder negativas, es decir que estuvieran basadas en el castigo para simbolizar la dinámica de subyugación sobre la pareja. El alfa de Cronbach de toda la escala reportado por Rivera et al. (2017) es de $\alpha=.63$. Para asegurar la confiabilidad del instrumento se realizaron evaluaciones de alfa de Cronbach por factor. El alfa de Cronbach en esta muestra para el factor de dominio es de $\alpha=.78$, mientras que para el factor de manipulación y chantaje es de $\alpha=.84$. Las respuestas en los reactivos de la escala de estrategias de poder van del uno al cinco.

El tercer instrumento utilizado es el de impulsividad estado, cuyos autores son Iribarren et al. (2011). La escala cuenta con tres factores: gratificación, automatismo y atención. Esta escala tiene un alfa de Cronbach de $\alpha=.88$, presentado por los autores de la escala. Se eligió la escala de impulsividad estado debido a que puede evaluar la impulsividad como una reacción comportamental ante un estímulo especifico, en este caso la pareja. Para asegurar la confiabilidad del instrumento se realizaron evaluaciones de alfa de Cronbach por factor. El factor de automatismo tiene un alfa de Cronbach de $\alpha=.82$, el factor de atención tiene un alfa de Cronbach de $\alpha=.85$, mientras que el factor de gratificación tiene un alfa de Cronbach de $\alpha$ $=.80$. Las respuestas en los reactivos en la escala de impulsividad estado van del cero a tres.

El cuarto instrumento utilizado fue la validación mexicana del inventario español de Estado, Rasgo y Expresión de Ira, de Oliva et al. (2010). Se eligió esta escala porque puede medir a la ira como rasgo, como estado y si es controlada o expresada. Este instrumento cuenta con 6 factores con un alfa de Cronbach de $\alpha$ $=.73$. Para asegurar la confiabilidad del instrumento se realizaron evaluaciones de alfa de Cronbach por factor. 
El factor de control externo de ira tiene un alfa de Cronbach de $\alpha=.92$. El factor de control interno de la ira tiene un alfa de Cronbach de $\alpha=.90$. El factor de expresión externa de la ira tiene un alfa de Cronbach de $\alpha=.76$. El factor de expresión interna de la ira tiene un alfa de Cronbach de $\alpha=.83$. El factor de sentimiento de ira tiene un alfa de Cronbach de $\alpha=.88$. El factor de temperamento iracundo tiene un alfa de Cronbach de $\alpha=.88$. El factor de deseos de expresar ira tiene un alfa de Cronbach de $\alpha=.83$. Por último, el factor de reacción de ira tiene un alfa de Cronbach de $\alpha=.81$. Las respuestas en los reactivos de la validación mexicana del inventario español de Estado, Rasgo y Expresión de Ira van del uno al cuatro.

\section{Procedimiento}

El investigador principal, así como asistentes contratados y capacitados para este fin encuestaron en lugares públicos de la ciudad de Puebla. En el momento en que se proporcionó el conjunto de escalas a responder, se entregó el consentimiento informado en el que se explicaba la confidencialidad de la información y se obtuvo la firma de consentimiento, también se cumplieron los otros requisitos éticos de la Asociación Americana de Psicología (Macomber, 2010).

\section{Análisis de datos}

Esta sección se dividió en tres etapas. La etapa uno consistió en correlaciones de Pearson en la que se midieron las relaciones entre la violencia psicológica/física, la violencia económica/social y la violencia sexual, con los factores de la ira, las estrategias de poder y los factores de la impulsividad. La etapa dos consiste en regresiones múltiples por pasos con la ira, las estrategias de poder y la impulsividad como predictores de la violencia psicológica/física, la violencia económica/social y la violencia sexual. Para la etapa tres se buscó cumplir el objetivo secundario de encontrar diferencias estadísticas entre los distintos tipos de violencia de pareja.

Se formaron grupos propósito con participantes que ejercieran la violencia de un nivel moderado a frecuente en: violencia económica/social, violencia psicológica/física y violencia sexual. Para formar los 
grupos se utilizó la mediana de las variables de violencia psicológica/física, violencia económica/social y violencia sexual para determinar qué participantes habían ejercido la violencia de manera moderada a frecuente y qué participantes habían ejercido la violencia de manera baja a moderada. Los grupos se consideraron de violencia moderada debido a la falta de participantes que ejercieran la violencia de manera frecuente.

Una vez conformados los grupos, se buscaron diferencias estadísticas entre estos en cuanto a la ira, la impulsividad y las estrategias de poder, utilizando la prueba de U-Mann Whitney. Como medida de control de los grupos, debían tener niveles moderados en un tipo de violencia y tener niveles bajos del tipo de violencia con el que contrastaban. Por ejemplo, se buscó la diferencia estadística entre el Grupo 1: violencia sexual moderada con violencia psicológica/física baja, con el Grupo 2: violencia psicológica/física moderada con violencia sexual baja. Los softwares que se utilizaron para todos los análisis estadísticos mencionados fueron SPSS 25 y G power 3.1.

\section{Resultados}

Primero es importante señalar que ninguno de los reactivos de las escalas tuvo más de $5 \%$ de datos perdidos. Los participantes con datos perdidos se suprimieron de la lista y se trabajó con el resto de los participantes. En la etapa 1 se realizaron correlaciones de Pearson entre las variables. En la Tabla 1 se presentan los resultados de las correlaciones.

\section{Tabla 1}

Tabla de correlaciones Pearson de la violencia de pareja psicológica/física, la violencia de pareja económica/social y la violencia sexual, con las estrategias de poder, la impulsividad y la ira

$\begin{array}{cccc}M D & \begin{array}{c}\text { Violencia } \\ \text { psicológica/física }\end{array} & \begin{array}{c}\text { Violencia } \\ \text { económica } \\ \text { /social }\end{array} & \begin{array}{c}\text { Violencia } \\ \text { sexual }\end{array} \\ \end{array}$

\section{Estrategias de poder}

Dominio

0.59

$.55 * *$

$.35^{* *}$


$\underline{\text { Impulsividad }}$

Automatismo

0.52

0.48

$.35 * *$

Es de

Atencional

0.69

0.52

$.30 * *$

$.20 * *$

$.14 * *$

Gratificación

0.47

0.44

$.18 * *$

$.17 * *$

.06

$.12 * *$ destacar que

.05

$\underline{\text { Ira }}$

Control externo de ira

2.37

0.75

$-.11 * *$

$-.02$

Control interno de ira

2.36

0.72

$-.05$

$-.02$

Expresión externa de ira 1.63

0.53

$.36 * *$

$.20 * *$

Expresión interna de ira

1.79

0.67

$.23 * *$

$.15^{* *}$

Temperamento iracundo

1.59

0.66

$.36^{* *}$

$.19 * *$

$.34 * *$

$.21 * *$

Deseos de expresar ira

$1.39 \quad 0.42$

Sentimiento de ira

$1.50 \quad 0.56$

$.33 * *$

$.21 * *$

$.36 * *$

$.30 * *$

Violencia

$1.82 \quad 0.61$

psicológica/física

$1.29 \quad 0.44$

$.62 * *$

Violencia

.98

0.42

$.62 * *$

1

económica/social

1.39

0.83

$.27 * *$

$.25 * *$

Nota. Valor a $p<.05$.

$1.62 * *$

las

Violencia sexual

1.39

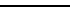

$.25 * *$

.003 estrategias de

$-.05$

$.08 *$ poder se

.08

.07 relacionaron

.08 con los tres

.06

.08 tipos de

$.27 * *$

violencia de

$25 * *$

pareja.

1 También se

señala que los

tres factores de la violencia de pareja se relacionan estadísticamente entre sí. En la etapa dos se presentan los resultados de las regresiones múltiples por tipo de violencia de pareja. Para generar el modelo predictor de la violencia psicológica/física se realizó una regresión múltiple por pasos. Se presenta la Tabla 2 con el modelo predictor de la violencia psicológica.

\section{Tabla 2}

Regresión múltiple por pasos de las estrategias de poder, la impulsividad y la ira como predictores de la violencia psicológica/física

\begin{tabular}{|c|c|c|c|c|c|c|c|c|c|c|}
\hline & $B$ & $S E B$ & B & $\Delta R^{2}$ & $R^{2}$ & $p$ & Tol. & VIF & $1-\beta$ & $f^{2}$ \\
\hline $\begin{array}{l}\text { Modelo } 1 \\
\text { Manipulación y } \\
\text { chantaje }\end{array}$ & .41 & .02 & .55 & .30 & .30 & $\begin{array}{l}.001 \\
.001\end{array}$ & 1 & 1 & 1 & .42 \\
\hline $\begin{array}{l}\text { Modelo } 2 \\
\text { Manipulación y } \\
\text { chantaje, dominio }\end{array}$ & $\begin{array}{l}.30 \\
.29\end{array}$ & $\begin{array}{l}.03 \\
.05\end{array}$ & $\begin{array}{l}.41 \\
.24\end{array}$ & .03 & .34 & $\begin{array}{l}.001 \\
.001 \\
.001\end{array}$ & $\begin{array}{l}.65 \\
.65\end{array}$ & $\begin{array}{l}1.53 \\
1.53\end{array}$ & 1 & .51 \\
\hline Modelo 3 & & & & .03 & .37 & .001 & & & 1 & .58 \\
\hline
\end{tabular}




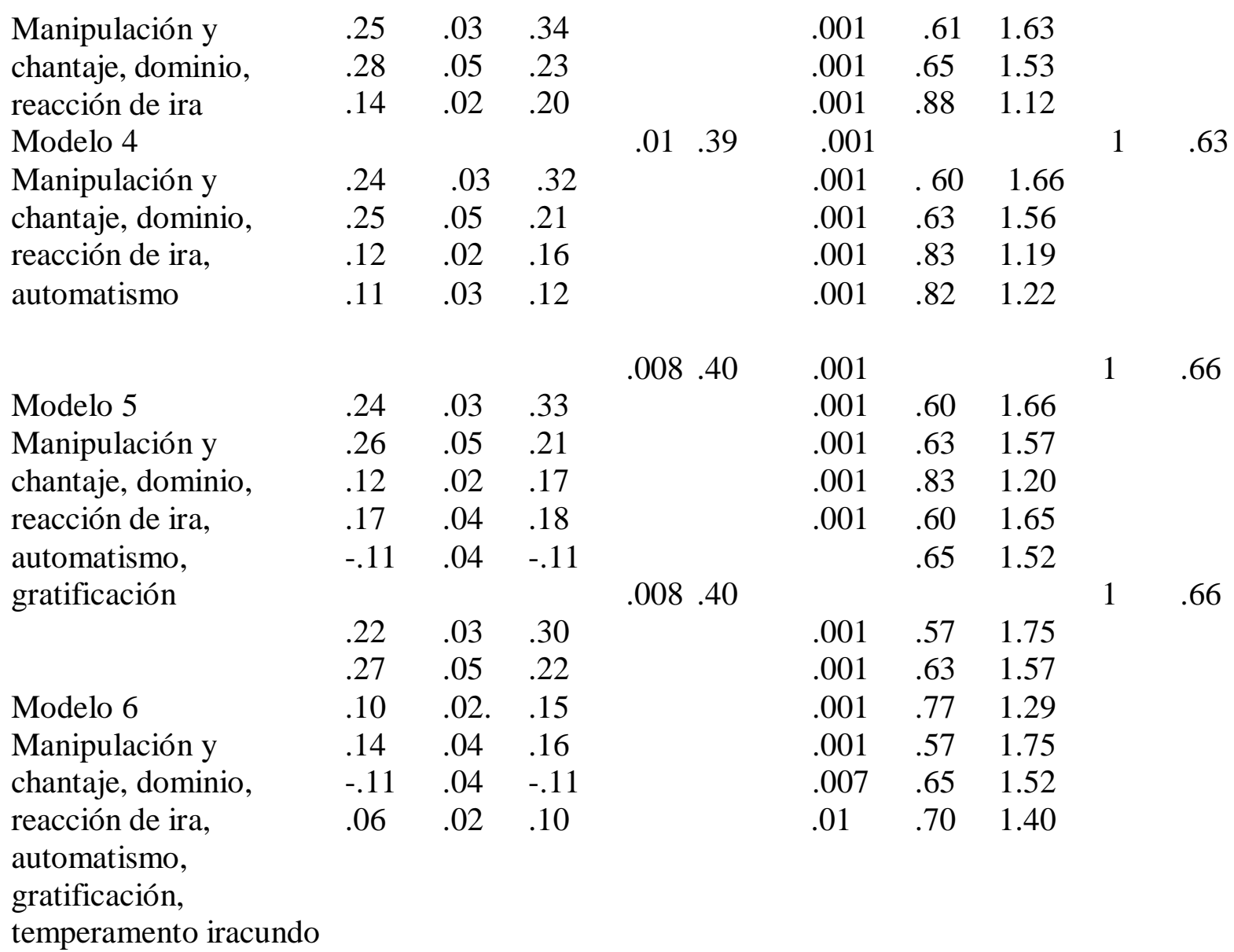

Nota. Valor a $p<.05$.

El sexto y último modelo predictor de la violencia psicológica/física tuvo una F de $(6,520)=59.82$, un tamaño del efecto de .66, que se considera alto, además de una evaluación de Durbin Watson de 1.75. También se buscó tener un modelo predictor de violencia económica/social, por lo que se realizó una regresión múltiple por pasos para poder contrastar en discusión los resultados con el modelo predictor de violencia psicológica/física. A continuación, se presenta la Tabla 3 con el resultado.

Tabla 3

Regresión múltiple por pasos de las estrategias de poder, la impulsividad y la ira como predictores de la violencia económica/social

\begin{tabular}{lcccccccccc}
\hline & $B$ & $S E B$ & $\mathrm{~B}$ & $\Delta R^{2}$ & $R^{2}$ & $P$ & Tol. & VIF & $1-\beta$ & $f^{2}$ \\
\hline $\begin{array}{l}\text { Modelo 1 } \\
\begin{array}{l}\text { Manipulación y } \\
\text { chantaje }\end{array}\end{array}$ & .24 & .02 & .35 & .12 & .12 & .001 & & & 1 & .13 \\
& & & & & & .001 & 1 & 1 & & \\
\end{tabular}




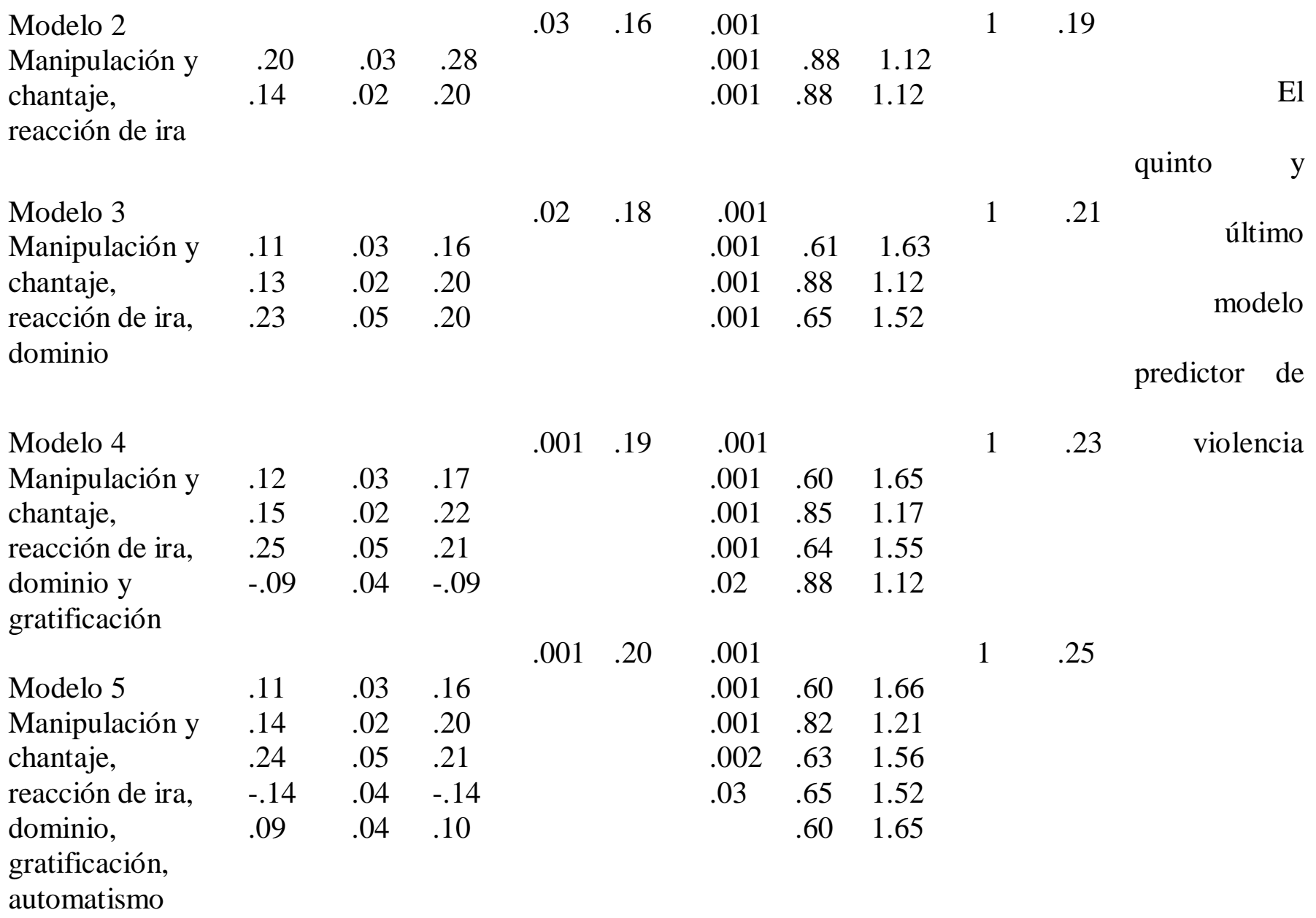

Nota. Valor a $p<.05$.

económica/social contó con una F de $(5,524)=26.90$, mientras que la evaluación de Durbin Watson fue de 1.44. El tamaño del efecto fue de .25 , que se consideró moderado. Se realizó un tercer modelo predictor, aunque esta vez fue del indicador de violencia sexual, para poder contrastar con el modelo de violencia psicológica/física y económica/social. Sin embargo, la regresión múltiple tuvo una evaluación de Durbin Watson de .54, por lo que se decidió realizar una regresión simple con cada una de las variables. A continuación, se presentan los resultados de las variables que fueron significativas (Tabla 4).

\section{Tabla 4}


Regresiones simples significativas de las estrategias de poder, la impulsividad y la ira como predictores de la violencia sexual.

\begin{tabular}{|c|c|c|c|c|c|c|c|c|c|}
\hline & $R$ & $R^{2}$ & $B$ & $S E B$ & $\beta$ & $F$ & $P$ & $1-\beta$ & $f^{2}$ \\
\hline \multicolumn{10}{|l|}{ Impulsividad } \\
\hline Automatismo & .14 & .02 & .24 & .07 & .14 & $(1,530) 10.89$ & .001 & .90 & .02 \\
\hline Atención & .12 & .016 & .20 & .06 & .12 & $(1,530) 8.71$ & .003 & .83 & .016 . \\
\hline $\begin{array}{l}\text { Estrategias de } \\
\text { poder }\end{array}$ & & & & & & & & & \\
\hline Dominio & .14 & .021 & .32 & .09 & 14 & $(1,530) 11.20$ & .001 & .92 & .02 . \\
\hline $\begin{array}{l}\text { Manipulación } \\
\text { y chantaje }\end{array}$ & .16 & .028 & .23 & .06 & .16 & $(1,530) 15.25$ & .001 & .97 & .02 \\
\hline
\end{tabular}

Nota. Valor a $p<.05$

Se señala nuevamente que las estrategias de poder fueron los mejores predictores junto con el factor de automatismo de la escala de impulsividad. Aunque la varianza explicada por los factores es muy baja. La etapa tres consistió en señalar las diferencias estadísticas en los niveles de presencia de la ira, la impulsividad y las estrategias de poder entre los factores que describen la violencia de pareja. Se hizo esto para contrastar con los resultados encontrados en las correlaciones y las regresiones múltiples en las que las variables elegidas no predecían mucho a la violencia sexual. Se presenta primero la evaluación de diferencia estadística del grupo de violencia sexual moderada con el grupo de violencia económica/social moderada, que se realizó con una evaluación de U-Mann Whitney. En la Tabla 5 se muestran las variables cuyas diferencias fueron significativas.

\section{Tabla 5}

Tabla de diferencias estadísticamente significativas con prueba de U-Mann

Whitney entre los grupos de la violencia sexual moderada y la violencia económica/social moderada.

\begin{tabular}{|c|c|c|c|c|c|c|c|c|c|}
\hline \multicolumn{3}{|c|}{$\begin{array}{l}\frac{\text { Violencia sexual moderada }}{\text { con violencia }} \\
\text { económica/social baja } n=60\end{array}$} & \multicolumn{3}{|c|}{$\frac{\text { Violencia económica/social }}{\frac{\text { moderada con violencia }}{\text { sexual baja } n=78}}$} & & & & \\
\hline Variables & $\begin{array}{r}\text { Rango } \\
\text { promedi } \\
0\end{array}$ & $\begin{array}{l}\text { Suma } \\
\text { de } \\
\text { rangos }\end{array}$ & $\begin{array}{c}\text { Rango } \\
\text { Promedio }\end{array}$ & $\begin{array}{l}\text { Suma } \\
\text { de } \\
\text { rangos }\end{array}$ & $\mathrm{Z}$ & $\mathrm{U}$ & $p$ & $1-\beta$ & $d$ \\
\hline $\begin{array}{l}\text { Manipulación } \\
\text { y chantaje }\end{array}$ & 56.93 & 3416 & 79.17 & 6175 & -3.24 & 1586 & .001 & .94 & .62 \\
\hline
\end{tabular}

Nota. Valor a $p<.05$ 
Se señala que existen mayores niveles de presencia en las variables de manipulación y chantaje en el grupo de violencia económica/social moderada $(M D=1.88$ y $D E=.61)$, en contraste con el grupo de violencia sexual moderada $(M D=1.55$ y $D E=.40)$. Para conocer las diferencias entre los grupos de violencia sexual moderada y violencia psicológica/ física moderada se realizó otra evaluación de U-Mann Whitney. Se presentan a continuación los resultados (Tabla 6).

\section{Tabla 6}

Tabla de diferencias estadísticamente significativas con U-Mann Whitney entre los grupos de la violencia sexual moderada y violencia psicológicalfísica moderada.

\begin{tabular}{|c|c|c|c|c|c|c|c|c|c|}
\hline & \multicolumn{2}{|c|}{$\begin{array}{l}\frac{\text { Violencia sexual }}{\text { moderada a alta con }} \\
\frac{\text { violencia }}{\text { psicológica/física }} \\
\text { baja }(n=31)\end{array}$} & \multicolumn{2}{|c|}{$\begin{array}{l}\frac{\text { Violencia }}{\text { psicológica/física }} \\
\frac{\text { moderada a alta con }}{\text { violencia sexual baja }} \\
\underline{(\mathrm{n}=95)}\end{array}$} & \multirow[b]{2}{*}{$\mathrm{Z}$} & \multirow[b]{2}{*}{$\mathrm{U}$} & \multirow[b]{2}{*}{$p$} & \multirow[b]{2}{*}{$1-\beta$} & \multirow[b]{2}{*}{$d$} \\
\hline & $\begin{array}{c}\text { Rango } \\
\text { promedi } \\
\text { o }\end{array}$ & $\begin{array}{c}\text { Suma de } \\
\text { rangos }\end{array}$ & $\begin{array}{c}\text { Rango } \\
\text { promedio }\end{array}$ & $\begin{array}{l}\text { Suma } \\
\text { de } \\
\text { rangos }\end{array}$ & & & & & \\
\hline $\begin{array}{l}\text { Man y } \\
\text { ch } \\
\text { Temp }\end{array}$ & 43.69 & 1354.50 & 69.96 & 6646.5 & -3.48 & 858.50 & .001 & .97 & .84 \\
\hline Ira & 44.84 & 1390 & 69.59 & 6611 & -3.28 & 894 & .001 & .88 & .67 \\
\hline
\end{tabular}

En el grupo de violencia sexual moderada existe una menor presencia de manipulación y chantaje $(M D=1.43$ y $D E=.43)$ y temperamento iracundo $(M D=1.35$ y $D E=.55)$, en contraste con el grupo de violencia psicológica/física moderada, que en la variable de manipulación y chantaje obtuvo una $M D=1.92$ y una $D E=.70$, mientras que la variable de temperamento iracundo obtuvo una $M D=1.77$ y una $D E=.68$. Por último, se realizó otra prueba de U-Mann Whitney entre los grupos propósito de violencia psicológica/física moderada y violencia económica/social moderada; sin embargo, no hubo diferencias estadísticamente significativas cuya potencia fuera aceptable. 


\section{Discusión}

Se cumplió el objetivo principal de generar modelos predictores para la violencia psicológica/física, económica/social y sexual. Los modelos predictores para la violencia psicológica/física y violencia económica/social muestran mujeres que viven con ira, no piensan antes de actuar y que de manera consistente tratan de controlar a su pareja, esto concuerda con teorías previas (Chester y DeWall, 2017; Dutton, 2007; Potter-Efron, 2015; Stark y Hester, 2019; Thornton et al., 2016).

Se señala que las estrategias de poder fueron los mejores predictores en todos los tipos de violencia de pareja. Esto sugiere lo importante que es el aprendizaje de comportamientos de control violento en la pareja para que la violencia ocurra. Una de las ventajas que ofrece la teoría general de la agresión es que propone que, sin importar si las personas están iracundas, queda en ellas el decidir si son violentas o no, lo cual es algo que se muestra en investigaciones recientes (Chester y DeWall, 2017; Gracia et al., 2017).

Por otro lado, es de destacar que las variables de la ira, la impulsividad y las estrategias de poder se relacionan menos, predicen menos y se encuentran menos presentes cuando se trata de violencia sexual. Esto coincide con otras investigaciones que no priorizan la ira o la impulsividad, aunque si el control-poder como causa de la violencia sexual femenina (Anderson et al., 2020; Conradi y Geffner, 2012; Krahé et al., 2003). Esto puede señalar una característica del poder en la pareja, el uso del sexo para controlar ya sea como castigo o negociación (Rivera y Díaz-Loving, 2002). Aunque el indicador de violencia sexual consiste en un solo reactivo, lo que se puede señalar como una limitación.

En cuanto a la violencia económica/social y psicológica/física, se señala que, si bien el tamaño del efecto en las regresiones múltiples es menor en la violencia económica/social, en sí no hay diferencia entre los dos factores en evaluación de prueba $t$. Agregando que, a su vez, la ira, las estrategias de poder y la 
impulsividad se han relacionado previamente con la violencia física, lleva a sugerir que la violencia económica, psicológica, social y física involucran el mismo proceso violento.

El hecho de que las mismas variables predigan los distintos tipos de violencia de pareja es algo que se encontró en los estudios de Conradi y Geffner (2012). Esto resulta digno de mención puesto que la violencia social o violencia económica pueden suceder sin el conflicto, mientras que la violencia física casi de manera inevitable sucede a la par de un conflicto. Además, se ha encontrado que la violencia física puede disminuir mientras que los demás tipos de violencia pueden aumentar (Krigel y Benjamin, 2020; Postmus et al., 2018). Esto señala que se tiene que trabajar aquello que provoca la violencia y no solamente en eliminar la violencia física, ya que la violencia se puede manifestar de distintas maneras.

También es importante mencionar que diversos autores señalaron que la creencia social de que el hombre es más violento que la mujer forma parte de los estereotipos que perpetuán la educación propia de la masculinidad tóxica (Frieze et al., 2020; Razera et al., 2017; Scarduzio et al., 2016). Por ello, educar que la mujer que es violenta con su pareja vive enojada y busca controlar a su pareja forma parte de un cambio cultural en cuanto al cómo se percibe el ser hombre y el ser mujer, porque se señala que la mujer violenta se comporta de manera similar al hombre violento y esto resulta en un acto que desafía los estereotipos de género y favorece conceptualizaciones de género más sanas.

Incluso diversos autores ya han señalado que mucha de la violencia de pareja es violencia mutua, un tipo de violencia en la que ambos se atacan el uno al otro (Conradi y Geffner, 2012; Johnson, 2008; Nicolson, 2019). Esto debe llevar a que se reconozca a la mujer no solo como víctima de la violencia sino también como perpetradora y por ende igual al hombre.

Entre las limitaciones que existen se señala que se tomó por igual a las relaciones de jóvenes mujeres de 18 años y las relaciones de mujeres adultas de más de 40 años, además de que se evalúan relaciones 
pasadas y presentes por igual. Aun así, la tendencia estadística se sostiene si se segmenta a la muestra por edades y estado civil, en datos que no se publican en esta investigación pero que se hicieron durante ella.

\section{Conflicto de intereses}

Los autores declaran no tener conflictos de intereses.

\section{Financiamiento}

El autor responsable le agradece al CONACYT el apoyo otorgado a través de la Beca para Estudios del doctorado en investigación psicológica \# 784252, del apoyo 452002.

\section{Referencias}

Alcázar-Córcoles, M., Verdejo, J. y Bouso-saiz, J. (2015). Propiedades psicométricas de la escala de impulsividad de Plutchik en una muestra de jóvenes hispanohablantes. Actas Españolas de Psiquiatría, 43(5), 161-169. https://www.actaspsiquiatria.es/repositorio/17/97/ESP/17-97-ESP161-9-505471.pdf

Bair-Merritt, M., Shea, S., Thompson, D., Sibinga, S., Trent, M. y Campbell, J. (2010). Why Do Women Use Intimate Partner Violence? A Systematic Review of Women's Motivations. Trauma Violence. Abuse, 11(4), 178-189. https://doi.org/10.1177/1524838010379003

Bandura, A. (1978). Social Learning Theory of Aggression. Journal of communication, 28(3), 12-29. https://doi.org/10.1111/j.1460-2466.1978.tb01621.x

Bewley, S. y Welch, J. (2014). ABC of domestic and sexual violence. Wiley Blackwell.

Birkley, E. y Eckhardt, C. (2015). Anger, hostility, internalizing negative emotions, and intimate partner violence perpetration: A meta-analytic review. Clinical psychology review, 37, 40-56. https://doi.org/10.1016/j.cpr.2015.01.002

Celma, J. (2015). Bases de teóricas y clínicas del comportamiento impulsivo. Ediciones San Juan de Dios. 
Cienfuegos, Y. (2014). Validación de dos escalas para evaluar violencia en la relación de pareja:

Perpetrador/a y Receptor/a. Revista de Psicología Iberoamericana, 22(1), 6271. https://www.researchgate.net/publication/295902592_Cienfuegos_Martinez_Y_2014_Validaci on_de_dos_escalas_para_evaluar_violencia_en_la_relacion_de_pareja_Perpetradora_y_Receptora _Revista_de_Psicologia_Iberoamericana_22_1_62_-71_ISSN_1405-0943.

Conradi, L, y Geffner, R. (2012). Female offenders of Intimate partner violence. Current Controversies, research and treatment approaches. Routledge.

Díaz-Loving, R., y Rivera, S. (2010). Antología psicosocial de la pareja, clásicos y contemporáneos. Porrúa/UNAM.

Dutton, D. (2006).Rethinking Domestic Violence. UBC Press.

Dutton, D. (2007). The abusive personality. Violence and control in intimate relationships. Guilford Press. Dutton, D. y Golant, S. (2012). El golpeador. Un perfil psicológico. Paidós.

Elias, L. y Saucier, D. (2006). Neuropsychology Clinical and experimental foundations. Pearson Education. Fernández, T., Martínez, F., Unzueta, C. y Rojas, É. (2016). Violencia hacia los varones entre parejas jóvenes universitarias de Tijuana, México. Enseñanza e Investigación en Psicología, 21(3), 255-263. https://www.redalyc.org/pdf/292/29248182005.pdf

Frankland, A. y Brown, J. (2014). Coercive control in same-sex intimate partner violence. Journal of Family Violence, 29(1), 15-22. https://doi.org/10.1007/s10896-013-9558-1

Frieze, I. H., Newhill, C. E. y Fusco, R. (2020). Dynamics of Family and Intimate Partner Violence. Springer Giordano, P., Copp, J., Longmore, M. y Manning, W. (2016). Anger, Control, and Intimate Partner Violence in Young Adulthood. Journal of family violence, 31(1), 1-13. https://doi.org/10.1007/s10896-0159753-3 
Iribarren, M., Jiménez-Giménez, M., García-de Cecilia, J. y Rubio-Valladolid., G. (2011). Validación y propiedades psicométricas de la Escala de Impulsividad Estado (EIE). Actas Españolas de Psiquiatría, 39(1), 49-60. https://actaspsiquiatria.es/repositorio/13/69/ESP/13-69-ESP-49-60341166.pdf

Johnson, M. (2008). A Typology of Domestic Violence: Intimate Terrorism, Violent Resistance, and Situational Couple Violence. Northeastern University Press.

Krahé, B., Waizenhöfer, E. y Möller, I. (2003). Women's Sexual Aggression Against Men: Prevalence and Predictors. Sex Roles, 49, 219-232. https://doi.org/10.1023/A:1024648106477

Krigel, K., Benjamin, O. (2020). From Physical Violence to Intensified Economic Abuse: Transitions Between the Types of IPV Over Survivors' Life Courses. Violence Against Woman. https://doi.org/10.1177\%2F1077801220940397

Lien, M. y Lorentzen, J. (2019). Men's experiences of violence in intimate relationships. Palgrave Macmillan.

López, M. (2013). Tratamiento psicológico de la impulsividad desde la perspectiva de las terapias de conducta de tercera generación. A propósito de un caso. Anuario de psicología / The UB Journal of psychology, 43(1), 117-127. https://www.redalyc.org/pdf/970/97027472008.pdf

Macomber, J. (2010). Publication manual of the American Psychological Association. APA.

McMurran, M., Blair,M. y Egan, V. (2002). An Investigation of the Correlations Between Aggression, Impulsiveness, Social Problem-Solving, and Alcohol Use. Aggressive behavior, 28, 439-445. https://doi.org/10.1002/ab.80017

Nedegaard, R. y Sbrocco, T. (2014). The Impact of Anger on the Intimate Partner Violence DecisionMaking Process. Journal of family violence, 29, 613-624. https://doi.org/10.1007/s10896-014-96181 
Nicolson, P. (2019). Domestic violence and psychology. Critical perspectives on intimate partner violence and abuse. Routledge.

Oliva, F., Calleja, N. y Hernández, M. (2010). Validación de la versión mexicana del inventario de expresión de ira estado-rasgo (STAXI-2). Acta colombiana de psicología, 13(2), 107-117. https://www.redalyc.org/pdf/798/79819279010.pdf.

Potter-Efron, R. (2015). Handbook of anger management and domestic violence offender treatment. Routledge.

Postmus, J. L., Hoge, G. L., Breckenridge, J., Sharp-Jeffs, N. y Chung, D. (2020). Economic Abuse as an Invisible Form of Domestic Violence: A Multicountry Review. Trauma, Violence, \& Abuse, 21(2), 261-283. https://doi.org/10.1177/1524838018764160

Razera, J., Gaspodini, I. B. y Falcke, D. (2017). Violencia marital y asimetría de género: una revisión integradora de la literatura. Psico-USF, 22(3), 401-412. http://doi.org/10.1590/141382712017220302

Resko, S. (2010). Intimate Partner Violence and Women's Economic Insecurity. LFB Scholarly Publishing LLC.

Rivera, S. y Díaz-Loving, R. (2002). La cultura del poder en la pareja. Porrúa.

Rivera, S., Díaz-Loving, R., Méndez, F., Jaen, C., García, M., Romero, A. y Villanueva, G. (2017). 8 escalas: el lado negativo de las relaciones de pareja. Manual Moderno.

Salvo, G. y Castro. A. (2013). Confiabilidad y validez de la escala de impulsividad de Barratt (BIS-11) en adolescentes. Revista Chilena de Neuropsiquiatría, 51(4), 245-254. https://doi.org/10.4067/S071792272013000400003. 
Scarduzio, J. A., Carlyle, K. E., Harris, K. L. y Savage, M. W. (2017). "Maybe She Was Provoked": Exploring Gender Stereotypes About Male and Female Perpetrators of Intimate Partner Violence. Violence Against Women, 23(1), 89-113. https://doi.org/10.1177/1077801216636240

Sears, A., Byers, E. y Price, E. (2007). The co-occurrence o adolescent boys' and girls' use of psychologically, physically and sexually abusive behaviors in their dating relationships. Journal of Adolescence, 30(3), 487-504. https://doi.org/10.1016/j.adolescence.2006.05.002

Siller, A., Trujano, P. y Velasco, S. (2012). Estudio sobre violencia doméstica en el DF: resultados preliminares. Nóesis, 22(44), 232- 255. https://doi.org/10.20983/noesis.2013.3.9

Snyder, L., Cho E. J. y Snyder, L. (2018). Intimate Partner Violence: A Bibliography of Theory, Research, and Intervention. Minnesota Center for Nonviolence.

Stark, E. y Hester, M. (2019). Coercive Control: Update and Review. Violence against Women, 25(1), 81104. https://doi.org/10.1177/1077801218816191

Thornton, A., Graham-Kevan, N. y Archer, J. (2016). Intimate Partner Violence: Are the Risk Factors Similar for Men and Women, and Similar to Other Types of Offending? Aggressive behavior, 42(4), 404-412. https://doi.org/10.1002/ab.21635

Teten, A., Schumacher, J., McLeish, A., Samper, R., McLeish, A. y Coffey, S. (2012). Relative Importance of Emotional Dysregulation, Hostility, and Impulsiveness in Predicting Intimate Partner Violence Perpetrated by Men in Alcohol Treatment. Psychology of Women Quarterly, 37(1), 51-60. https://doi.org/10.1177/0361684312461138

Williams, J., Ghandour, R. y Kub, J. (2008). Female perpetration of violence in heterosexual intimate relationships: adolescence through adulthood. Trauma Violence Abuse, 9(4), 227-249. https://doi.org/10.1177/1524838008324418 
Cómo citar este artículo: Galindo Moto, M. A. ., Cruz del Castillo, C. ., Lechuga Paredes, R. E. ., Díaz-Loving, R. .., Romero Palencia, A. ., \& Padilla Bautista, J. A. . (2021). La impulsividad, la ira y las estrategias de poder como predictores de la violencia de pareja, con mujeres perpetradoras. Psicumex, 11(1), 1-25, e357. https://doi.org/10.36793/psicumex.v11i1.357 